\title{
Assessment of diet in young children with an emphasis on fruit and vegetable intake: using CADET - Child and Diet Evaluation Tool
}

\author{
JE Cade*, L Frear and DC Greenwood \\ Nutritional Epidemiology Group, Centre for Epidemiology and Biostatistics, University of Leeds, 30-32 Hyde \\ Terrace, Leeds LS2 9LN, UK
}

Submitted 17 January 2005: Accepted 31 August 2005

\begin{abstract}
Objectives: To develop a concise, simple tool for use by non-specialists to assess diet in children aged 3-7 years attending primary schools.

Design: A 24-hour food tick list covering all aspects of the diet and with a focus on fruit and vegetable consumption was developed. This was compared against a 24-hour semi-weighed food diary obtained for the same day as the tick list.

Setting: Six primary schools with a range of socio-economic and ethnic backgrounds from a large city in the north of England (Leeds).

Participants: One hundred and eighty children returned completed packs of information; a response rate of $77 \%$ of those who were willing to take part, $48 \%$ of those approached.

Results: On average, 2.4 items of fruit including juice ( 2.1 items as 5-a-day count) were eaten and 1.6 items of vegetables (excluding potato). Twenty-seven per cent and 36\% of boys and $23 \%$ and $24 \%$ of girls reported not eating any fruit or vegetables, respectively, on the recording day. Correlations comparing the diary and tick list were high for both foods (range $r=0.44$ to 0.89 ) and nutrients (range $r=0.41$ to 0.68 ). The level of misclassification was much less than would be expected by chance. Parent and teacher evaluation of the tick list was very positive. Parents felt the tick list was easy and quick to complete.

Conclusion: The Child and Diet Evaluation Tool (CADET) tick list has been used successfully for rapid collection of food and nutrient information from children aged 3-7 years from diverse social and ethnic backgrounds. The tool has performed better than many food-frequency questionnaires in comparison to a food diary.
\end{abstract}

The promotion of healthy eating should start from the early stages of life ${ }^{1}$. Schools provide a highly effective and efficient way to reach children, staff, families and community members ${ }^{1}$. Children have low dietary intake of fruits and vegetables amounting to around $2-3$ servings per day ${ }^{2}$ and one in five children eat no fruit at all during a week $^{3}$. Assessment of diet in young children is particularly challenging and presents specific problems for the research team ${ }^{4}$. Until the age when children become aware of their food intake and can conceptualise time (at about 8 years old), parents are relied upon to report the diet of their children. Parental reports of young children's diets using questionnaire methods may be accurate enough to be useful in nutritional screening and dietary surveillance of fruit and vegetable intake 5 . However, as with adult diet, children's reported diet is prone to measurement error.

The National School Fruit Scheme aims to increase the intake of fruit and vegetables in children aged 4 to 7 years. In order to evaluate this scheme, an effective and easily administered tool is necessary to assess the diet of this population prior to and during this intervention. The tool must be simple for use with large numbers, easy to complete and analyse. Previous studies regarding dietary assessment in children highlight strengths and weaknesses in a range of tools ${ }^{6,7}$. Currently there is no suitable tool that can be used by a non-specialist to evaluate the diets of young children in the UK. The aim of the present research was to develop such a tool.

\section{Method}

\section{Recruitment of schools and subjects}

Six state primary schools, representative of the different social classes and ethnic backgrounds in Leeds, a large city in the north of England, were selected to participate in the study. Three schools were involved in the national Sustain Grab 5 programme promoting fruit in schools, with one school being chosen randomly from each level of intervention. This ensured that the tool was tested on schools with a wide range of fruit availability and consumption. 
Each school included two classes chosen at random: Nursery, Reception, Year 1 or Year 2. Each class contained on average 25 children; hence there were 12 classes in total making approximately 300 children in the study. We aimed to recruit 300 children aged 3 to 7 years for the validation study, which would allow for a drop-out rate of $25 \%$. Schools were offered a contribution (£2.00/child taking part) towards school funds as thanks for their co-operation.

\section{Sample size}

A total sample of 230 children, each completing the new method and the 'gold standard' tool, would give $80 \%$ power to detect an average bias of a third of a portion of combined fruit and vegetable intake, using the conventional 5\% significance level. A third of a portion is less than $10 \%$ of the recommended 5 portions of fruit and vegetables and would therefore give close agreement on average.

\section{Dietary assessment method}

\section{4-Hour tick list questionnaire (CADET)}

The Child and Diet Evaluation Tool (CADET) was developed to be used prospectively as a tick list record for all foods consumed over one 24-hour period, with a retrospective breakfast section.

The tick list had three parts. The part for completion at home by the parent or caregiver included 92 foods (including 12 fruits, 10 vegetables, four beans/seeds) and seven drinks (one fruit juice) for each of four meal/snack events. The part for completion at school lunchtime by a dinner supervisor or parent classroom assistant included 82 possible foods eaten at lunchtime and seven drinks. The third part was for use in the class immediately after break times; this pictorially displayed 20 snack food and seven drink options including nine fruits and vegetables and fruit juice for the children to mark as instructed by the classroom teacher.

The tool required only ticks for the items consumed in the relevant boxes, with no necessity to record quantity or weight of the food/drink item, and was therefore quick to complete. The 24-hour tick list was completed during or at the end of each meal/snack event except for breakfast, which was undertaken retrospectively by the parent. Time spent collecting data from each child was limited to a few minutes following each break time.

\section{Comparison method}

The method used for comparison with the 24-hour tick list was a prospective 1-day semi-weighed food diary. The diary was administered on the same day as the tick list and covered the same period of time. As with the 24-hour tick list the food diary had two sections: one for completion at school and the other for completion at home.
In the home section, the parent/carer was required to complete a detailed record of all food and drink consumed by the child at home. Items were either weighed (home scales) or measured by household portions (estimated). Breakfast was completed retrospectively as with the tick list. The diaries were returned to the research team the following day and then checked for completeness. Parents were telephoned if necessary for more detail.

For the school section, a researcher recorded in detail food eaten during the school day for each child. Directly prior to the children being served school dinners, the research team weighed each food and drink option. These weights were then applied to the diary with leftovers described and estimated. The researcher also recorded break-time snacks following completion of the CADET break-time form by the children. The researcher spoke to each child individually immediately after they had filled in the pictorial form to determine which snacks were consumed.

\section{Repeatability}

In order to assess repeatability information was collected from a subgroup of two classes of children on two different days, data collected one week apart. A second food diary was also obtained with the repeat CADET tool.

\section{Data coding}

The tick lists and food diaries were coded and analysed using a Microsoft ${ }^{\circledR}$ Access-based food diary analysis program (DANTE). This program was designed by the Nutrition Epidemiology Group at the University of Leeds particularly for research using food diaries and incorporates a recipe analysis function as well as very flexible data handling and output. Double data entry was used. Portion sizes used in the analysis of the tick list were based on mean food intakes from the National Diet and Nutrition Survey (NDNS) results for children ${ }^{3}$. These portion sizes were age- and gender-specific.

\section{Statistical analysis}

Results from the two methods were compared in terms of limits of agreement and overall bias using Bland-Altman plots $^{8,9}$. Correlation coefficients determined any significant correlations between the tick list and food diary. Paired $t$-tests assessed significant differences between the two methods of assessment. These statistics were applied for all children combined, and separately for boys and girls and for different ages.

\section{Ethical committee approval}

Approval for the study was obtained from the local research ethics committee in Leeds. Permission was also sought for the study from the headteacher and governing body of each school involved. Positive parental consent was obtained. 


\section{Results}

\section{Response rate}

Of the 375 children approached to take part in the study, $67 \%$ (251) responded. Of these, 93\% (234) were willing to take part in the study. One hundred and ninety-two children returned packs, of which 180 were filled in correctly. This is a response rate of $77 \%$ of those who were willing to take part, $48 \%$ of those approached.

The schools taking part included a range of different backgrounds and areas as illustrated by the free school meal index, which ranged from $4 \%$ to $28 \%$ of children receiving free school meals.

Parent's evaluation of the tick list was very positive in terms of ease of completion. The tick list was rated as easy to understand by $73 \%$ of parents and as not at all time consuming by $51 \%$. Only $2 \%$ reported it was difficult and only $1 \%$ of children reported not liking it. Teachers found the project caused very little disruption to normal classroom activity. Indeed, they felt that the tool had potential as a learning experience for the children.

\section{Sample and bousebold characteristics}

The sample obtained consisted of 56\% (100) boys and 44\% (80) girls. The mean age of the children was 4.8 years. Table 1 shows further sample characteristics. About 10\% of children lived in households with one adult and about $17 \%$ of children were the only child in the household. Of the children who responded, 5\% of their households contained people without any academic qualifications and about 25\% were from households where someone had a degree. About 90\% were from households where someone had a paid job.

The majority of the children were from households which classified themselves as white European origin.

Table 1 Sample characteristics

\begin{tabular}{lcc}
\hline & Boys $(n=100)$ & Girls $(n=80)$ \\
\hline Mean age (years) & 4.7 & 4.9 \\
Age distribution (\%) & 21 & 14 \\
3 years & 27 & 24 \\
4 years & 22 & 30 \\
5 years & 19 & 23 \\
6 years & 11 & 10 \\
7 years & 12 & 8 \\
One adult in household (\%) & 16 & 18 \\
One child in household (\%) & & \\
Qualifications in household (\%) & 5 & 5 \\
$\quad$ None & 28 & 23 \\
Degree & 88 & 92 \\
Paid job in household (\%) & & \\
Household ethnicity (\%) & 83 & 90 \\
$\quad$ White & 2 & 0 \\
Indian & 7 & 1 \\
Pakistani & 2 & 3 \\
Black Caribbean & 1 & 0 \\
Chinese & 5 & 6 \\
Other & & \\
\hline
\end{tabular}

Nine per cent of the boys and 1\% of the girls were from households of Indian or Pakistani origin.

Results from the tick list show that $4 \%$ of boys and $2 \%$ of girls reported having no breakfast. However, most (62\%) of the children ate breakfast cereal during the day. Again from the tick list, most children were consuming full-cream milk (54\%), 34\% consumed semi-skimmed milk and $4 \%$ were consuming skimmed milk. Six per cent of children did not have any milk on the day of recording. There were differences in milk intake by age for boys, with more of the 7-year-old boys drinking no milk compared with younger boys. Most children (84\%) ate some bread/rolls/toast during the day with the majority (61\%) eating one or two slices. White bread was most popular (59\%), 9\% were eating white with added fibre and 9\% were eating wholemeal bread. Fat spreads were used by the majority: 22\% butter or butter-type spreads, $14 \%$ soft margarine, $11 \%$ polyunsaturated margarine, $7 \%$ olive spread, 14\% low-fat spread and 13\% did not have any fat spread. Seventy-six per cent of children did not have sugar added to food or drinks, although 3\% were eating 7 or more teaspoons over the day. Seventeen per cent of the children were given a dietary supplement on the day of recording.

\section{Food intake}

Comparison of tick list and food diary

The tick list gave somewhat higher mean portions for fruit and a lower mean value for total vegetables recorded over the day (Table 2). Mean values are for the whole sample and not just consumers. In particular, the tick list gave higher values for fruit juice than the diary. Individual fruits varied in their agreement with the tick list, providing similar weights of portions consumed for bananas but giving higher weights for apples/pears and oranges than the diary. Fifty-seven per cent of children were correctly classified for number of portions of vegetables (excluding beans) and also fruit (excluding juice) by the tick list compared with the diary. The mean difference between the tick list and diary for total fruit and vegetables was $45 \mathrm{~g} \mathrm{day}^{-1}$; however, limits of agreement were wide.

Correlation coefficients, although not assessing agreement, did indicate that children were ranked in a similar fashion and were all highly statistically significant, ranging from 0.44 for fruit juice to 0.89 for baked beans. Girls tended to have slightly higher correlations than boys for the items considered. Exploration of the data according to age of the child showed little difference.

\section{Fruit and vegetables}

Cross-check questions on the CADET asked for the number of servings of fruit and vegetables in total consumed during the day. Twenty-seven per cent of boys and 23\% of girls were reported as not eating any fruit on the day of recording and $36 \%$ of boys and $24 \%$ of girls did 
not eat any vegetables (excluding potatoes). All occurrences of fruit or vegetables eaten during the day were counted from the CADET responses. The mean number of items of fruit, including fruit juice, was 2.4; excluding fruit juice, 1.6. When the 5-a-day count was used (i.e. fruit juice counted only once) the number of items was 2.1. The mean number of vegetable items eaten during the day on the tick list was 1.6; if beans and pulses are excluded from this count then the value drops to 1.4 items. There was no difference between boys and girls in the numbers of items of fruit or vegetables consumed.

The most popular types of fruit were apple/pear ( $\sim 40 \%)$, bananas $(\sim 30 \%)$ and orange/satsuma types ( $\sim 20 \%)$. The most popular vegetables were carrots, peas and other salad (not tomato). About 20\% of children ate baked beans on the day of recording.

\section{Nutrients}

\section{Comparison of tick list and food diary}

The tick list was compared against the 1-day diary for nutrient intakes. These are compared in Table 3. On average the tick list gave slightly higher values for nutrient intakes than the diary, except for polyunsaturated fat and total carotene. The differences were within $10 \%$ of the mean values for fat, saturated fat, monounsaturated fat, polyunsaturated fat, calcium, carotene and vitamin C. The paired $t$-test or Wilcoxon signed rank sum test showed that these absolute differences between the two methods were statistically significant except for calcium, saturated and polyunsaturated fat and carotene. However, although statistically significant, the differences were small. The two methods were correlated and showed a close association for most of the nutrients. In particular, there was a strong association for fibre, carotene and folate between the two methods. Girls had higher correlation coefficients than boys for energy, carbohydrate, fat, calcium and iron, and boys had higher coefficients for protein, fibre and vitamin C.

Nutrient intakes increased with age of the child. The trend of increase in nutrient intake with age was more pronounced for the diary results than for those from the tick list. For each age, energy intake from the tick list was higher than from the diary. However, for vitamin $\mathrm{C}$ the tick list gave higher values for the younger children and lower values for the older children than the diary.

The tick list tended to give the lowest values for nutrients for the schools with a high free school meal index (i.e. the poorer schools) and the highest values for schools with a low free school meal index (i.e. the better-off schools). Differences between schools according to free school meal index were statistically significant for fibre and vitamin C (Kruskal-Wallis test). The diary also tended to give lower values for nutrients for the schools with the highest school meal index. In general, these differences were larger than for the tick list and all 
Table 3 Comparison of nutrients between the tick list and diary

\begin{tabular}{|c|c|c|c|c|c|c|}
\hline & \multicolumn{2}{|c|}{ Tick list } & \multicolumn{2}{|c|}{ Diary } & \multirow[b]{2}{*}{ Difference $\left(P\right.$-value $\left.{ }^{\star}\right)$} & \multirow[b]{2}{*}{ Correlation coefficient ( $P$-value $)$} \\
\hline & Mean & $\mathrm{SD}$ & Mean & $\mathrm{SD}$ & & \\
\hline \multicolumn{7}{|l|}{ Energy } \\
\hline (MJ) & 7.5 & 1.7 & 6.5 & 1.8 & 1.0 & \\
\hline (kcal) & 1800 & 411 & 1563 & 433 & $242(<0.0001)$ & $0.43(<0.0001)$ \\
\hline Protein (g) & 58 & 14 & 50 & 15 & $8(<0.0001)$ & $0.41(<0.0001)$ \\
\hline Carbohydrate (g) & 260 & 62 & 220 & 68 & $40(<0.0001)$ & $0.41(<0.0001)$ \\
\hline Fat $(\mathrm{g})$ & 66 & 19 & 60 & 20 & $6(<0.0001)$ & $0.46(<0.0001)$ \\
\hline Saturated fat (g) & 24.6 & 7.5 & 23.7 & 9.2 & $0.9(0.07)$ & $0.41(<0.0001)$ \\
\hline Monounsaturated fat (g) & 19.1 & 5.7 & 18.1 & 6.8 & $1(0.03)$ & $0.43(<0.0001)$ \\
\hline Polyunsaturated fat $(\mathrm{g})$ & 7.9 & 2.7 & 8.0 & 3.7 & $-0.1(0.99)$ & $0.32(<0.0001)$ \\
\hline Fibre, Englyst (g) & 10.1 & 3.2 & 8.4 & 3.8 & $1.7(<0.0001)$ & $0.59(<0.0001)$ \\
\hline Calcium (mg) & 847 & 258 & 838 & 360 & $9(0.72)$ & $0.43(<0.0001)$ \\
\hline Iron (mg) & 9.1 & 2.7 & 7.7 & 3.4 & $1.4(<0.0001)$ & $0.43(<0.0001)$ \\
\hline Carotene $(\mu \mathrm{g})$ & 1330 & 1293 & 1420 & 1783 & $-90(0.85)$ & $0.68(<0.0001)$ \\
\hline Folate (mg) & 195 & 59 & 172 & 80 & $23(<0.0001)$ & $0.52(<0.0001)$ \\
\hline Vitamin C (mg) & 93.3 & 49.9 & 86.8 & 80.4 & $6.5(0.003)$ & $0.46(<0.0001)$ \\
\hline
\end{tabular}

SD - standard deviation.

${ }^{*}$ Differences tested using the paired $t$-test or Wilcoxon signed rank test as appropriate.

differences tested were statistically significant. However, the highest values were not necessarily from schools with the lowest free school meal index.

\section{Bland-Altman plots}

Another approach to assessing the agreement between two dietary assessment methods is to explore Bland-Altman plots, which plot the mean values of nutrients from the two methods against the differences between the two methods. In general, the plots showed a reasonably wide spread of values, as shown by the standard deviation (SD) lines, although the difference was quite close to 0. Examples of plots for energy, fat, fibre and vitamin $\mathrm{C}$ are shown in Fig. 1.

\section{Classification by tertiles}

A final test of the validity of the tick list nutrients compared with the diary nutrient values was carried out by dividing each nutrient into tertiles and exploring the degree of misclassification which occurred by tertile. The highest percentage of children classified into the same tertile was for fibre (57\%) and the lowest was for energy (43\%). These are all greater than would be expected to occur by chance (33\%). Twelve per cent were grossly misclassified according to protein intake and only $7 \%$ for calcium. This level of misclassification is much less than expected by chance.

\section{Repeatability}

Thirty children completed two CADET tools; of these 27 also had a repeat diary. The first CADET gave higher results by about $10 \%$ than the second CADET. The second CADET gave values slightly closer to the second diary, implying a learning effect on completing the CADET. The first CADET did not correlate particularly well with the second CADET; however, these were from two different days and would not be expected to correlate as well with each other as with the relevant diary that was taken on the same day as the CADET.

\section{Discussion}

We have developed a dietary assessment tool which is quick, simple and easy to complete by non-specialists. Food and nutrient intakes from the tick list appear to be somewhat higher compared with values from a semiweighed food diary.

The CADET study was representative of the school-aged (3-7 years) population of Leeds. The Leeds school-aged population, as part of the Yorkshire and Humber region, has $11.3 \%$ from ethnic minorities and $18 \%$ entitled to free school meals. Nationally, $17.1 \%$ of primary school-aged children are entitled to free school meals and $13.6 \%$ are from ethnic minorities ${ }^{10}$. Results of the CADET evaluation study show a similar distribution with $10 \%$ of the sample population from ethnic minorities and $15.2 \%$ entitled to free school meals.

A third of boys and a quarter of girls reported eating no fruit or vegetables at all on the study day. This result reflects a similar finding of one in five children not eating any fruit in any one week ${ }^{11}$. In a randomised controlled trial to reduce obesity risk factors of children aged between 7 and 11 years, the subjects reported eating 1.8 portions of fruit and 0.5 portion of vegetables ${ }^{12}$. These findings are again similar to those from the CADET study and are alarmingly low in amount. The NDNS has shown that 4-6-year-old boys consume on average $155 \mathrm{~g}$ fruit (excluding juice) and $60 \mathrm{~g}$ vegetables (excluding potatoes) daily; $157 \mathrm{~g}$ and $58 \mathrm{~g}$, respectively, for girls. Using data from the tick list of popular fruit and vegetables in this study, a mean of $255 \mathrm{~g}$ was consumed. The current recommendation is 5 servings of fruit and vegetables $(400 \mathrm{~g})$ per day (excluding potatoes) ${ }^{3}$. However, these 
506

(a)

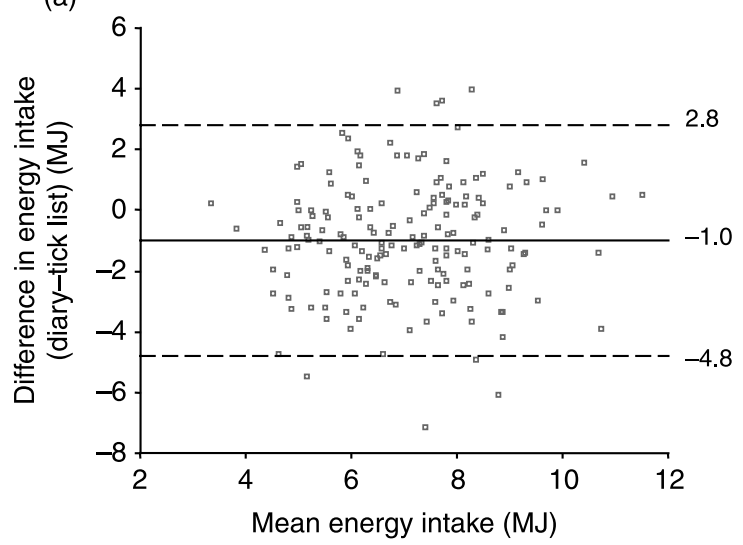

(c)

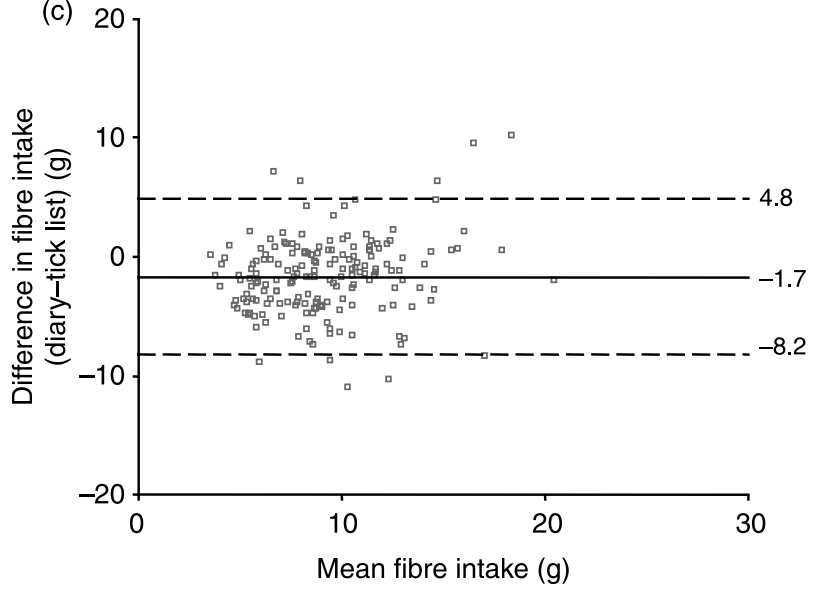

(b)

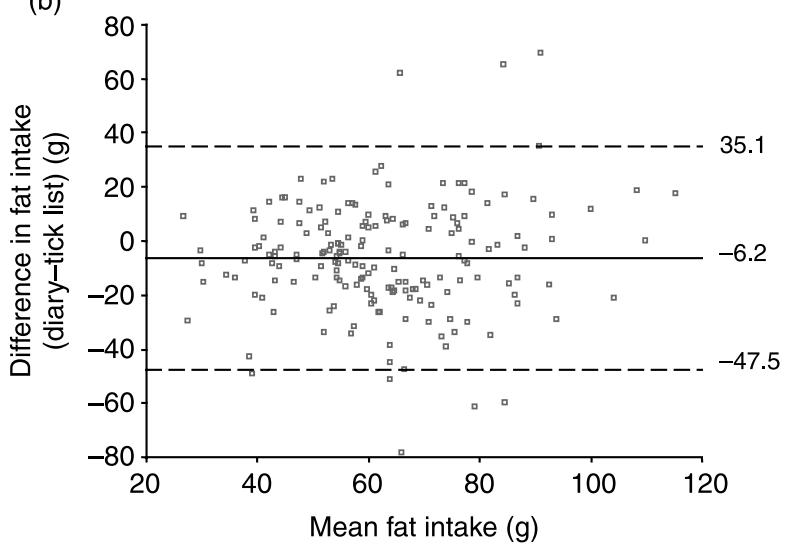

(d)

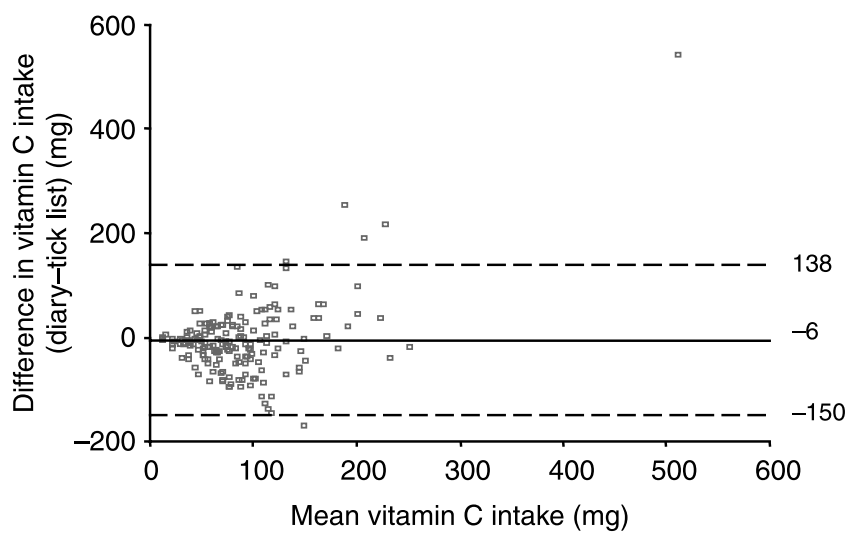

Fig. 1 Agreement between the diary and tick list (Bland-Altman plots) for (a) energy, (b) fat, (c) fibre and (d) vitamin C. Upper and lower dashed lines indicate limits of agreement (i.e. $\pm 2 S D$ ); middle solid line indicates the mean difference between the methods (SD, standard deviation)

recommendations are for an adult population and should be adjusted to allow for age and gender.

The most popular fruits consumed in the study were apples/pears, bananas and oranges/satsumas. This is obviously a reflection not only of taste but also of seasonality, cost and shelf-life ${ }^{13}$. Popular vegetables were carrots, peas, salad and baked beans. Approximately half of the children studied reported consuming pure fruit juice on the study day, making an important contribution to total fruit consumption. Vegetable intake was lower than fruit intake. It is clear that there is a considerable need to increase both fruit and vegetable intake within this age group for both genders ${ }^{3}$.

The tick list generally gave higher values than the food diary for fruit intake, with higher numbers of portions for fruit juice being recorded than on the diary. This could possibly be due to incorrect classification of fruit juice on the tick list, with some participants regarding fruit drinks as fruit juices. It is also possible that drinks are more frequently forgotten when completing a food diary ${ }^{14,15}$ as opposed to working through a tick list with prompts to aid memory.

The mean difference in total fruit and vegetable consumption was $45 \mathrm{~g}$, which is rather small, although the limits of agreement $( \pm 2$ SD) were wide $(-327$ to $417 \mathrm{~g} \mathrm{day}^{-1}$ ). This indicates that the tick list may slightly overestimate intakes compared with a diary. However, there is no true 'gold standard' and food diaries tend to underestimate intakes. The small sample included in the repeatability analysis indicates that the tick list may be useful for assessing change in intake.

There are two key aspects regarding the CADET tool which need to be considered: first, the ease of use of the tool for this age group of children (discussed below) and second the appropriateness of the portion sizes employed to generate the amounts of food consumed and nutrient totals. Portion sizes for the CADET were provided from the $\mathrm{NDNS}^{3}$; however, for some foods the number of children in specific age/gender groups was small, leading potentially to unreliable portion estimates. For example, the portion size for porridge for boys aged 6 years was based on a sample of one child. Even for something as commonly consumed as hard cheese, the portion for 7-year-old girls was based on a sample of only 27 children. To further illustrate the point, an alternative analysis of the CADET tool using other published portion size data ${ }^{16}$ resulted in lower nutrient values for the tick list than the 
diary (data not shown). For future use of the tool it will be possible to adjust portion sizes to be appropriate to the target age/gender group.

\section{Reliable assessment of nutrients}

Most food-frequency questionnaires (FFQs) have a tendency to overestimate ${ }^{17}$. The CADET does however overestimate less than is expected from other FFQs and correlates better. Comparison of the absolute differences in nutrient intakes between the two methods showed some statistically significant differences. These differences were small, amounting at worst to $18 \%$ of the mean nutrient intake for fibre and $17 \%$ for carbohydrate. Nutrient information and amounts of food/drink consumed are dependent not only on information provided by the participant but also on the portion sizes assigned to the food/drink items ${ }^{18}$. In this case age- and genderspecific NDNS portion sizes appear to overestimate some items. This overestimation is, however, substantially lower than that of most FFQs ${ }^{19}$ and explains the differences with the food diary for all nutrients tested. Ongoing work will adjust these portion sizes; initial analysis has shown even stronger correlations and reduction in the differences between methods.

The CADET resulted in a strong correlation for nutrients ranging from 0.41 to 0.68 , which is equivalent to or better than that of most other food frequency-type questionnaires which on average correlate at 0.3 to 0.4 compared with weighed intakes ${ }^{17}$. The CADET was compared with a food diary recorded on the same day and therefore the two methods could not be entirely independent. However, to avoid potential bias, wherever possible the CADET was completed by school staff and the food diary was completed by the fieldwork team. For meals eaten at home, both tools had to be completed by the parent/carer.

Many studies agree on the 'suitability' of the FFQ for recording dietary intake, suggesting that short, inexpensive measures that assess dietary intake can be as responsive as multiple-day diet records ${ }^{19-22}$. A US study of 97 children aged 6-10 years concluded that parental reports of young children's diets using food frequency methods are accurate compared with biomarkers, and can be used in fruit and vegetable intake analysis and nutritional evaluation ${ }^{5}$. Biomarkers were not available to us for further validation of the CADET.

The CADET gives similar mean nutrient intakes to those obtained from children aged 4-6 years in the NDNS (4-18 years). Vitamin $\mathrm{C}$ and fibre intakes are higher for girls than for boys in both the tick list and diary. This is possibly due to a recorded higher intake of fruit and vegetables for girls, affecting these nutrients. This was also a finding of the $\mathrm{NDNS}^{3}$, which also showed a lower intake for boys of high-fibre cereals which could also be another factor affecting their lower fibre intake.

Correlations were higher for girls than boys, suggesting parental/carer attitude and knowledge regarding girl's diets to be more accurate, possibly through greater interest or possibly a greater overestimation of intake for boys than for girls. This further explains the difference in nutrient totals for the tick list compared with the diary ${ }^{3,17,19}$.

\section{Practical for quick collection from large groups}

The tick list proved practical for quick coding in large samples. No training of school staff or parents was required for completing the tick list. This method avoids the known limitations of obtaining dietary details from children by self-report ${ }^{23,24}$.

Measuring diets of children to support and evaluate nutritional interventions can be a difficult task. In our study we relied on parents and teachers to complete the tick list since details from such young children may be unreliable. Use of the pictorial check list by the children following breaks confirmed the snack and drink consumption that had been observed by the research team. In fact, the youngest children were generally provided with the break-time snacks by the school and so this was relatively straightforward to assess.

The FFQ approach may not be suitable for assessment of all foods or nutrients in children. In this case, a 1-day tick list may not reflect true longer-term intake. However, in spite of important reservations, it may be a useful tool to provide a rapid, simple assessment in a large population when more time-consuming, resource-demanding or complicated methods cannot be used ${ }^{19}$.

\section{Conclusion}

The CADET tick list has been used successfully for rapid collection of food and nutrient information from children aged 3-7 years. The tool has performed better than many FFQs in comparison to a food diary. It is now being used for a full evaluation of the National School Fruit and Vegetable Scheme.

\section{Acknowledgements}

We would like to thank the following people for help with the study: our collaborators Dr Mary Rudolf and Dr Pinki Sahota for support during the early stages; the fieldwork team, Beverley Bratley, Emma Elliot, Gavin McArt, Carol Levine, Shiela Sive and Michelle Spence; and our project assistant Karen Lawson. Thanks also to Karen Barton and Dr Wendy Wrieden from the University of Dundee for providing the NDNS portion size data. The work was funded by the Department of Health.

\section{References}

1 Perez-Rodrigo C, Klepp KI, Yngve A, Sjostrom M, Stockley L, Aranceta J. The school setting: an opportunity for the implementation of dietary guidelines. Public Health Nutrition 2001; 4: 717-24. 
2 Gibson EL, Wardle J, Watts CJ. Fruit and vegetable consumption, nutritional knowledge and beliefs in mothers and children. Appetite 1998; 31: 205-28.

3 Gregory J, Lowe S, Bates CJ, Prentice A, Jackson L, Smithers G, et al. National Diet and Nutrition Survey: Young People aged 4 to 18 years. London: The Stationery Office, 2000.

4 Livingstone MBE, Robson PJ, Wallace JMW. Issues in dietary intake assessment of children and adolescents. British Journal of Nutrition 2004; 92: S213-22.

5 Byers T, Trieber F, Gunter E, Coates R, Sowell A, Leonard S, et al. The accuracy of parental reports of their children's intake of fruits and vegetables: validation of a food frequency questionnaire with serum levels of carotenoids and vitamins C, A, and E. Epidemiology 1993; 4: 350-5.

6 Coufopoulos A-M, Maggs C, Hackett A. Doing dietary research with adolescents: the problems of data collection in the school setting. International Journal of Health Promotion E Education 2001; 39: 100-5.

7 McPherson RS, Hoelscher DM, Alexander M, Scanlon KS, Serdula MK. Dietary assessment methods among schoolaged children: validity and reliability. Preventive Medicine 2000; 31: S11-33.

8 Bland JM, Altman DG. Comparing two methods of clinical measurement: a personal history. International Journal of Epidemiology 1995; 24: S7-14.

9 Bland JM, Altman DG. Statistical methods for assessing agreement between two methods of clinical measurement. Lancet 1986; 1: 307-10

10 Department for Education and Skills. Statistics of Education Schools in England 2002 Edition. London: The Stationery Office, 2002.

11 Chinn S, Rona RJ. Prevalence and trends in overweight and obesity in three cross sectional studies of British children, 1974-94. British Medical Journal 2001; 322: 24-6.

12 Sahota P, Rudolf MC, Dixey R, Hill AJ, Barth JH, Cade J Randomised controlled trial of primary school based intervention to reduce risk factors for obesity. British Medical Journal 2001; 323: 1029-32.

13 Department of Health. The National School Fruit Scheme: Evaluation Summary. London: The Stationery
Office, 2001. Also available at http://www.dh.gov.uk/assetroot/04/01/92/32/04019232.pdf.

14 Barasi ME. Human Nutrition. A Health Perspective. London: Arnold, 1997.

15 Garrow JS, James WPT, Ralph A. Human Nutrition and Dietetics. London: Churchill Livingstone, 2000.

16 Ministry of Agriculture, Fisheries and Food. Food Portion Sizes, 2nd ed. London: The Stationery Office, 1993.

17 Cade J, Thompson R, Burley V, Warm D. Development, validation and utilisation of food-frequency questionnaires. Public Health Nutrition 2002; 5: 567-87.

18 Shimizu H, Ohwaki A, Kurisu Y, Takatsuka N, Ido M Kawakami $\mathrm{N}$, et al. Validity and reproducibility of a quantitative food frequency questionnaire for a cohort study in Japan. Japanese Journal of Clinical Oncology 1999; 29: $38-44$

19 Blom L, Lundmark K, Dahlquist G, Persson LA. Estimating children's eating habits. Validity of a questionnaire measuring food frequency compared to a 7-day record. Acta Paediatrica Scandinavica 1989; 78: 858-64.

20 Kristal AR, Beresford SA, Lazovich D. Assessing change in diet-intervention research. American Journal Clinical Nutrition 1994; 59: 185S-9S.

21 Clapp JA, McPherson RS, Reed DB, Hsi BP. Comparison of a food frequency questionnaire using reported vs standard portion sizes for classifying individuals according to nutrient intake. Journal of the American Dietetic Association 1991; 91: $316-20$.

22 Rockett HR, Colditz GA. Assessing diets of children and adolescents. American Journal of Clinical Nutrition 1997; 65: $1116 \mathrm{~S}-22 \mathrm{~S}$

23 Domel SB, Baranowski T, Davis H, Leonard SB, Riley P, Baranowski J. Fruit and vegetable food frequencies by fourth and fifth grade students: validity and reliability. Journal of the American College of Nutrition 1994; 13: 33-9.

24 Lemaitre RN, King IB, Patterson RE, Psaty BM, Kestin M, Heckbert SR. Assessment of trans-fatty acid intake with a food frequency questionnaire and validation with adipose tissue levels of trans-fatty acids. American Journal of Epidemiology 1998; 148: 1085-93. 